\title{
ABSTRACTS OF PAPERS
}

\section{SUBMITTED FOR PRESENTATION TO THIS SOCIETY}

The following papers have been submitted to the Secretary and the Associate Secretaries of the Society for presentation at meetings of the Society. They are numbered serially throughout this volume.* Cross-references to them in the reports of the meetings will give the number of this volume, the number of this issue, and the serial number of the abstract. $\dagger$

\section{Dr. A. A. Albert: The structure of pure Riemann matrices} with non-commutative multiplication algebras.

The chief outstanding problem in the theory of Riemann matrices is the determination of the structure of all pure Riemann matrices $\omega$ with a given non-commutative division algebra $B$ as multiplication algebra $D$. The problem is here reduced essentially to the case where $B$ is a normal division algebra. We consider in detail the case where $B$ is a known normal division algebra, that is, an algebra in $n^{2}$ units and of type $R_{n}$. In that case we find not only the structure of $\omega$ but that of its principal matrix, and obtain necessary and sufficient conditions on $D$ that it be a multiplication algebra of some $\omega$, and the form of the $\omega$ in terms of a single arbitrary submatrix. The theory is applied to the cases of generalized quaternion algebras, generalized quaternion algebras over a quadratic field, and to Cecioni algebras. In the first two cases actual classes of numerical examples of Riemınn matrices are given, these being the first known examples of Riemann matrices with non-commutative division multiplication algebras.

2. Dr. A. A. Albert: A construction of rational cyclic division algebras of order sixteen.

As is known, all normal division algebras in sixteen units are Cecioni algebras. However, the only algebras of order sixteen which have been constructed are the few isolated algebras of Cecioni. We consider here

* In the future, abstracts of papers will appear under the heading given above, separately from the reports of meetings, as explained in the announcement of pages 1-2 of this issue. Eventually, many abstracts will be printed in advance of the meeting at which the corresponding paper is presented, but this will not be done until the abstracts of papers that have been read have been printed.

$\dagger$ Thus, if an abstract numbered 238 is printed in issue No. 5 of volume 36, the cross-reference will be (Abstract No. 36-5-238). 
the class of algebras called cyclic (Dickson) algebras in sixteen units, a subset of the set of all decioni algebras. The known conditions that such algebras be division algebras are merely sufficient, and have not been shown to be necessary. We find necessary and sufficient conditions that such algebras be division algebras, and, by applying our theory to the case of algebras over the field of all rational numbers, construct an infinity of cyclic algebras for every cyclic quartic in the canonical form

$$
\omega^{4}+2 \nu\left(\Delta_{1}{ }^{2}+\Delta_{2}^{2}\right) \omega^{2}+\nu^{2} \Delta_{2}{ }^{2}\left(\Delta_{1}{ }^{2}+\Delta_{2}^{2}\right)=0,
$$

with $\nu$ a product of distinct rational primes, $\Delta_{1}$ and $\Delta_{2}$ relatively prime, $\Delta_{1}{ }^{2}+\Delta_{2}{ }^{2}=\sigma^{2} \rho$ with $\rho>1$ a product of distinct rational prime integers.

\section{Mr. D. H. Lehmer: On numbers associated with the divisors of an integer.}

Associated with every positive integer $n$ is its set of distinct divisors, which we designate by $\delta_{1}$. There is also a set of divisors associated with each number of the set $\delta_{1}$. In this way the set $\delta_{1}$ gives rise to a new set $\delta_{2}$ of numbers (not all distinct) which are in fact the divisors of the divisors of $n$ itself. The set $\delta_{2}$ generates in the same way a set $\delta_{3}$ and so on. The elements of the set $\delta_{r}$ we call the $r$ th divisors of $n$. If we take proper divisors instead of divisors we obtain similarly a set $d_{r}$ which we call the set of $r$ th proper divisors of $n$. The study of the connection between the same arbitrary function of $\delta_{r}$ and $d_{r}$ leads to certain inversion formulas which are applied to the determination of formulas for the product and sum of the $k$ th powers of the $r$ th divisors of $n$. The above definition of $\delta_{r}$ is meaningless if $r$ is negative, but if negative values of $r$ are used in the formulas for the above sums we obtain a sequence of functions of $n$ among which are Euler's totient function $\phi(n)$ and Merten's inversion function $\mu(n)$, or more generally Jordan's function $J_{k}(n)$. The present investigation can be considered either as a generalization of Jordan's function, or as a study of the sequence of functions $f_{r}(n)$ connected by the relation $f_{r+s}(n)=\sum f_{s}\left(\delta_{r}\right)$, where $f_{0}(n)=n^{k}$.

\section{Mr. D. H. Lehmer: A generalization of Lucas' functions} $U_{n}$ and $V_{n}$.

The functions $U_{n}=\left(a^{n}-b^{n}\right) /(a-b)$ and $V_{n}=a^{n}+b^{n}$, where $a$ and $b$ are the roots of $x^{2} \times P x+Q=0,(p, Q)=1$, have been studied by Lucas, Carmichael, and others. It is shown in this paper that a more homogeneous theory results if $P$ is replaced by $R,(R, Q)=1$, and if $U_{n}$ and $V_{n}$ are considered as functions of $R$ and $Q$. Although $U_{n}$ and $V_{n}$ may contain the factor $R$ in this theory, nearly all of Lucas' theorems apply with a slight modification. Some parts of the present theory, however, have no counterpart in Lucas' theory. Laws governing the appearance and repetition of a prime in the series $U$ are similar to those of Lucas. In developing these laws one is led to a new generalization of Fermat's theorem and Euler's totient function. New generalizations of Wilson's theorem result from a discussion of certain special cases of the series $U$. Associated with $U_{n}$ expressed as a function of $R$ and $Q$ is a certain irreducible binary form $G_{n}$ which represents numbers whose factors 
are of the forms $k n \pm 1$. The connection between $G_{n}$ and Sylvester's cyclotomic functions is established. A transformation is discussed which replaces $a$ and $b$ by their $r$ th powers. The inverse of this transformation leads to a new extension of the theory of quadratic and higher residues. This new theory is applied to a discovery of necessary and sufficient conditions for the primality of large numbers of certain types.

5. Dr. C. C. Craig (National Research Fellow): The semiinvariants and moments of incomplete normal and Type III frequency functions.

The answers to the following questions depend on a knowledge of the moments or of the semi-invariants of incomplete frequency functions. (1) How can a truncated frequency distribution be completed? (2) The method commonly used for finding the moments or semi-invariants of an index, $y / x$, is really valid only if the range of $x$ is limited; how do results obtained in this way differ from those in which the range for $x$ is unrestricted? (3) How would the characteristics of the best possible sample from an infinite population differ from those of the parent? To the previous results in the case of the incomplete normal frequency function, the present paper adds direct expressions for the semi-invariants, a simple recursion formula for the moments, and expressions for the moments in polynomials with the range as argument. The Type III curve of Pearson is similarly studied. The direct expressions for the semiinvariants are expressed by a kind of generalized Laguerre polynomials involving the range and skewness in the argument. Simple recursion relations for the moments are found. Numerical examples and selected tables are appended.

\section{Mr. W. L. Langdon and Professor Oystein Ore: Shep- pard's correction and semi-invariants.}

The use of semi-invariants for the characterisation of statistical distributions has for many applications a distinct advantage. This paper deals with the problem of correcting semi-invariants, and a very simple formula for the corrected semi-invariants has been established.

7. Mr. W. R. Thompson: The powers of a given rational prime which may divide exactly the discriminant of an algebraic field of nth degree.

For a given rational prime, $p$, Ore (Mathematische Annalen, vol. 96 (1926), pp. 313-352) has shown, in a given algebraic field of $n$th degree whose discriminant is $d$, the dependence upon the prime ideal decomposition of $p$ of the exponent, $E$, such that $d$ is exactly divisible by $p^{E}$. He has given the maximum value of $E$ for such a field which he designates by $N(n, p)$. He suggests the interest of discovering what other values $E$ may have. The author presents the following complete solution of this problem. Let $E_{p}{ }^{(n)}$ be the set of possible values of $E$ for fields of $n$th degree, and $\alpha$ be a positive rational integer. Then for $p>2$ and $n=p^{\alpha}, E_{p}^{(n)}=0, \cdots, N(n, p)$ except 
$\alpha p^{\alpha-1}-1$ for $\alpha>1, p>2$, and $n=p^{\alpha}+1, E_{p}^{(n)}=E_{p}{ }^{(n-2)}$, for $p>2$ in every other case, $E_{p}{ }^{(n)}=0 \cdots,(N, p)$, and if $p=2$ the result is the same as the above except that the number 1 is never a component of $E_{2}{ }^{(n)}$.

\section{Dr. A. K. Mitchell: The derivation of tensors from tensor} unctions.

In this paper, the author first outlines the method by which E. Schroedinger (Annalen der Physik, vol. 82 (1927), p. 265) obtains a rule for deriving a tensor from an invariant tensor function. It is pointed out that Schroedinger's proof applies only for euclidean space. A proof, holding for any metric, is then given of the theorem that if $\phi$ is any invariant function of a tensor its derivative with respect to a component of this tensor is itself the component of a tensor, and also that the derivative of a tensor function of a tensor produces a tensor of higher rank. In the second part of the paper this theorem is applied to the invariants of a mixed tensor of rank 2. The invariants of these derived tensors are found as functions of the original invariants. Finally the theorem is used to give immediate proofs of some of the elementary facts in algebraic invariant theory, as that the discriminant of an $n$-ary quadratic form is an invariant of weight 2 .

9. Mr. Samuel Borofsky: Expansion into infinite products of functions defined by Dirichlet series.

In this paper, it is proved that a function defined in a half-plane by a Dirichlet series

$$
1+b_{1} e^{-\mu_{1} z}+\cdots+b_{n} e^{-\mu_{n} z}+\cdots,
$$

where $\lim \sup \left(\log n / \mu_{n}\right)$ is finite, can be expressed in some half-plane as an infinite product $\pi\left(1+a_{n} e^{-\lambda_{n} z}\right)$, where $0<\lambda_{1}<\cdots<\lambda_{n}<\cdots, \lim \lambda_{n}=\infty$, and $\lim \sup \left(\log n / / \lambda_{n}\right)$ is finite, the $\lambda$ 's being linear combinations of the $\mu$ 's with positive integral coefficients. This generalizes the corresponding theorem of Ritt for power series.

10. Mr. F. H. Miller: An application of the method of parameters to linear partial differential equations.

In this paper it is shown that the method of variation of parameters, widely employed in determining the complete primitive of a linear ordinary differential equation whose complementary function is known, may also be used in connection with certain linear partial differential equations of the second order. Conditions under which the method is applicable are considered, and the connection between it and the usual Laplace transformation is discussed.

11. Professor J. F. Ritt: On a certain ring of functions of two variables.

We deal with functions (1): $a_{1}(x) \alpha_{1}(y)+\cdots+a_{n}(x) \alpha_{n}(y)$, with the $a$ 's and $\alpha$ 's analytic, but otherwise arbitrary. The product of two functions of this type is a function of similar type. One may thus propose to establish 
a factorization theory for functions (1). It is understood, in what follows, that the function; (1) are so expressed that the $a$ 's in them, and also the $\alpha$ 's, are linearly independent. It is proved that if $A=B C$, where $A, B, C$ are of type (1), every $a$ in $B$ or $C$ is an algebraic combination of the $a$ 's in $A$, if one makes exception of an arbitrary multiplicative function; similarly for the $\alpha$ 's. It follows that a function $a(x) \alpha(y)$ cannot be the product of two functions (1) each of which has more than one term. Also, the general factorization problem becomes a problem in algebraic functions of several variables. It is shown that $1+x y+\phi(x) \psi(y)$, with $\phi$ and $\psi$ transcendental, is not the product of two functions (1), each of more than one term.

12. Professors Philip Franklin and C. L. E. Moore: Systems of linear partial differential equations.

In this paper the authors discuss the solution of a pair of linear partial differential equations of the second order, and obtain analytically a result of Segre's, that the geometric interpretation of the solution is either a locus in 3-space, or the developable surface of a twisted curve. Some extensions to other systems are made.

13. Professor J. D. Tamarkin: A lemma of the theory of differential systems.

In this paper, the author states and proves a new lemma on linear differential systems with Lebesgue integrable coefficients, from which results that have been given by certain other authors can be derived in a simple manner. In particular, the results of Miss Whelan (this Bulletin, vol. 35(1929), pp. 105-125), and the results of a paper by W. M. Whyburn (see this Bulletin, vol. 36 (1930), p. 59), can be derived from the lemma. This paper and that of W. M. Whyburn will appear in the February issue of this Bulletin.

\section{Dr. P. M. Swingle: Generalized indecomposable continua.}

In this paper two of the more useful definitions of indecomposable continua are generalized. A number of theorems are proved concerning the sets thus defined, and the impossibility of generalizing another of these definitions in an obvious manner is shown.

15. Dr. Jesse Douglas: Various forms of the fundamental Junctional in the problem of Plateau and its relation to the area functional.

The writer's work on the problem of Plateau (see abstracts in this Bulletin, vol. 33 (1927), pp. 143, 259; vol. 34 (1928), p. 405; vol. 35 (1929), p. 292) is based on considering all possible representations $x_{i}=g_{i}(\theta) ; i=1$, $2 \cdots, n$; of the given contour as topological image of the unit circle, and reducing the problem to finding the representation $g$ which minimizes the functional 


$$
A(g)=-\frac{1}{2 \pi} \int_{0}^{2 \pi} \int_{0}^{2 \pi} \log \left|\sin \frac{1}{2}(\theta-\phi)\right| \sum_{i} d g_{i}(\phi) .
$$

In the present paper the following new forms are found for $A(g)$ :

$$
\begin{aligned}
& A(g)=\frac{1}{16 \pi} \int_{0}^{2 \pi} \int_{0}^{2 \pi} \sum_{i}\left[g_{i}(\theta)-g_{i}(\phi)\right]^{2}\left[\sin \frac{1}{2}(\theta-\phi)\right]^{-2} d \theta d \phi \\
& A(g)=\frac{\pi}{2} \sum_{m=1}^{\infty} m\left(\sum_{i} a_{i m}^{2}+\sum_{i} b_{i m}^{2}\right)
\end{aligned}
$$

where $a_{i m}$ and $b_{i m}$ denote the Fourier coefficients of $g_{i}(\theta)$. Sufficient conditions are established for the convergence of the infinite processes involved. These formulas prove $A(g)$ to be always positive. The form (2) subsumes the problem under the theory, initiated by Hilbert, of quadratic forms in a denumerable infinity of variables. The functional $A(g)$ is different from the area of the harmonic surface determined by the boundary values $g_{i}(\theta)$. However, these two functionals take their minimum values for the same representation $g$, and these minimum values are equal. Thus the functional $A(g)$ serves the same purpose as the area functional while it is decidedly simpler of expression and treatment. The specific advantage of the author's method in the problem of Plateau lies precisely in the substitution of $A(\mathrm{~g})$ for the more complicated area functional.

\section{Dr. Jesse Douglas: A general formulation of the problem of Plateau.}

In this paper methods are developed for treating the problem of Plateau in the following general formulation. Given $k$ contours in n-dimensional euclidean space. To determine a minimal surface bounded by the $k$ contours (and with no other boundaries) and having a prescribed topological structure, that is, genus and two-sidedness or one-sidedness. This minimal surface is, further, to be represented as a conformal image of a canonical configuration of the same topological structure. The canonical configuration may always be represented as a region bounded by circles; we consider all possible ways of representing the contours as topological images of these circles:

$$
x_{i}=g_{i}^{(1)}(\theta), x_{i}=g_{i}^{(2)}(\theta), \cdots, x_{i}=g_{i}{ }^{(k)}(\theta) .
$$

The problem is then reduced to the minimization of a certain functional $A\left(g^{(1)}, g^{(2)}, \cdots, g^{(k)}\right)$, generalizing $A(g)$ of the previous paper. The expression for this functional involves, in the place of the trigonometric functions occurring in $A(g)$, those functions which belong to a Riemann surface of the topological structure prescribed for the required minimal surface. For instance, for a minimal surface bounded by two given contours and to be represented conformally on a circular ring, the expression for $A\left(g^{(1)}, g^{(2)}\right)$ involves elliptic functions, having periods $2 \pi, 2 p(-1)^{1 / 2}$, where $p$ denotes the logarithm of the ratio of the radii of the circular ring. The existence of the required minimal surface depends on the existence of a real root for a certain definite equation in $p$. These ideas extend to the general case, where the moduli of Riemann intervene in place of the parameter $p$. 


\section{Professor O. E. Glenn: Integral invariants and secular perturbations.}

It is proved in this paper that any planetary orbit perturbed from the true elliptical form by attractions of other planets moves upon a trajectory which may be regarded as the free orbit of a properly determined central force. The transformations which give the perturbed orbit from the corresponding ellipse are made the basis of a theory of perturbations considered from the standpoint of integral invariants.

\section{Dr. T. H. Gronwall: A formula in geometrical optics.}

It is shown that the double surface integral giving the total radiation from a surface $S$ upon a surface $S_{1}$ may be transformed into a double line integral over the boundaries $C$ and $C_{1}$. In the special case where $S$ and $S_{1}$ lie in two parallel planes, the limit of the integral when the planes coincide is $\pi A$, where $A$ is the area which $S$ and $S_{1}$ have in common when superposed.

\section{Professor James Pierpont: On Cayley's definition of non- euclidean geometry.}

In his sixth memoir on quantics (1859), Cayley incidentally laid the foundations of a projective definition of distance and angle. Whether he saw the relation of his work to that of Lobachevsky and Bolyai is not known. At any rate, the foundations for a simple and complete theory of noneuclidean geometry (in the older sense) are there. The author has developed the implications of this paper, and wishes to show that this treatment of the subject is superior in some if not in all ways to the traditional method of Klein.

\section{Professor Edward Kasner: The $\infty^{3}$ plane sections of an arbitrary surface.}

The plane sections of an arbitrary surface form a triply infinite system of importance in projective differential geometry. By projection (orthogonal or central) on a fixed plane a related system is obtained whose characteristic geometric properties are studied. The first property, that the focal locus is always a circle, is the same as for dynamical trajectories (see the author's Princeton Colloquium Lectures, p. 10, or Transactions of this Society, vol. 7 (1906), p. 405), but the others are quite different. The $\infty^{4}$ spherical sections of a surface, fundamental in inversion geometry, are discussed briefly.

\section{Professor W. A. Wilson: On the Phragmén-Brouwer theorem.}

The purpose of this note is to give an elementary demonstration of the Phragmen-Brouwer theorem, for spaces other than the euclidean plane, which does not require a knowledge of the theory of dimensionality as a basis. The theorem is shown to be true in any metric space satisfying these requirements: (a) every bounded set has at least one limiting point; (b) if $a$ and $b$ are any two 
points, $d$ is the distance between them, and $k$ is any positive number less than $d$, then there is one and only one point $c$ whose distances from $a$ and $b$, respectively, are $k$ and $d-k$. The proof is based upon the validity of the theorem in . the euclidean plane.

22. Professor W. A. Wilson: A property of certain hyperspaces.

Let $M$ be a metric space and $H$ the hyperspace whose points or elements are the bounded closed sets of $M$, with the distance between any two elements defined as in Hausdorff's Mengenlehre (pp. 145-150). This paper shows that, if every bounded set in $M$ has at least one limiting point and $M$ is everywhere locally connected, then $H$ enjoys these properties. Consequently we have, as a generalization of the well known space filling curves, the result that the bounded closed sets of $n$-dimensional euclidean space (or an $n$-dimensional closed sphere) may be put in a continuous correspondence with the points of a euclidean ray (or segment).

23. Mr. J. M. Feld and Mr. Philip Newman: On the representation of analytic functions of several variables as infinite products.

The object of this paper is to prove (1) that an analytic function

$$
f(x, y)=1+\sum b_{m n} x^{m} y^{n}
$$

can be uniquely represented as an absolutely convergent infinite product $\Pi\left(1+a_{m n} x^{m} y^{n}\right)$ with constant $a$ 's, and (2) that $f(x, y)$ can be represented in the form $\Pi_{1}{ }^{\infty}\left(1+P_{n}\right)$, where $P_{n}$ is a homogeneous polynomial in $x$ and $y$ of degree $n$.

24. Dr. L. W. Cohen (National Research Fellow): A remark on a system of equations with infinitely many unknowns.

The non-singular system of equations $x_{i}+\sum_{k} a_{i k} x_{k}=y_{i}$, where

$$
\sum_{i}\left[\sum_{k}\left|a_{i k}\right|^{p /(p-1)}\right]^{p-1} \quad \text { and } \quad \sum_{i}\left|y_{i}\right|^{p}
$$

converge $(p>1)$, has a unique solution $\left\{x_{k}\right\}$ such that $\sum_{k}\left|x_{k}\right|^{p}$ converges. It is shown that the inverse of this transformation is of the same type, that is,

$$
\sum_{i}\left[\sum_{k}\left|\left({ }^{k} k\right)\right| p /(p-1)\right]^{p-1}
$$

converges where $\left({ }^{i} k\right)$ is the minor of $a_{i k}$.

25. Dr. A. B. Brown (National Research Fellow): Relations between the critical points and curves of a real analytic function of two independent variables.

In this paper the case where both isolated critical points and curves of critical points appear is treated for the first time by precise methods. Two inequalities and one equation are obtained, involving the isolated points and curves of minimum and maximum, and the "critical points of intermediate type." 
26. Professor C. J. Coe: Exterior motion in the restricted problem of three bodies.

This paper treats of that case of the restricted problem of three bodies in which the particle moves outside of the closed outer oval of zero relative velccity. Necessary and sufficient conditions are first established that the outer oval be closed, both for a given ratio of the two finite masses and independently of this ratio. The exterior orbit of the particle is first discussed for the neighborhood of its points of contact with the closed outer oval, the nature of these cusps in the rotating plane being completely determined. Next are developed two different sets of sufficient conditions that the particle recede to infinity, and an extension is made of Koopman's discussion of the areal velocity in fixed space for distant portions of the plane. There follow four theorems treating of the angular velocity of the particle, the most striking result being that for orbits not extending to infinity, the motion in the rotating plane can be direct only within a narrow ring surrounding the closed outer oval. Following this are proved five theorems on the total angular displacement of the particle showing, for instance, that it can never advance as much as $60^{\circ}$ in the rotating plane.

\section{Professor G. A. Parkinson: Pairs of curves in an $S_{n}$.}

This paper discusses pairs of curves in a flat $n$-dimensional space. In the first part of the paper it is shown that all of the more important properties of Bertrand curves carry over from euclidean 3-space to this more general space, either directly or in slightly modified form. In the subsequent parts of the paper are discussed pairs of curves which have a common pth $(p>2)$ normal; pairs of curves for which the principal normal of one lies in the osculating plane of the other; pairs of curves for which the principal normal of one lies in the plane of the principal normal and binormal of the other; and involutes of curves.

\section{Professor H. M. Gehman: Centers of symmetry in analysis situs.}

In this paper is given first a definition of a center of symmetry (in the sense of analysis situs) of a point set lying in an $n$-dimensional euclidean space. A property which holds true in this case is then used to define a center of symmetry of a point set when the set itself is considered as a space. The principal result of the paper is a characterization of a simple continuous arc from $A$ to $B$ as a closed (or a regular) point set $M$ containing $A$ and $B$ and at least one more point, such that (1) every point of $M-A-B$ is a center of symmetry of $M$, and (2) $A$ is a non-cut point of $M$. This definition of an arc, like Sierpinski's, does not explicitly assume that the set $M$ is connected.

29. Mr. N. C. Fisk: An investigation of surfaces in euclidean 4-space by means of 3-vectors. Preliminary report.

The properties of a surface in 3-space may be investigated by studying the change in the tangent plane corresponding to an arbitrary displacement 
on the surface. In this case the tangent plane is uniquely represented by the unit normal vector. The tangent plane of a surface in 4 -space may be represented uniquely by a pair of unit 3-vectors, each in a separate 3-space. This paper studies the properties of a surface in 4-space by investigating the changes in each of a pair of unit 3-vectors corresponding to an arbitrary displacement on the surface. The changes in the unit 3-vectors give rise to a pair of quadratic differential forms, the generalization of the second fundamental form for a surface in 3-space. From the quadratic forms four scalar invariants are obtained.

30. Professor H. T. Davis and Mr. V. V. Latshaw: Polynominal approximation by the method of least squares.

This paper, following a suggestion of Karl Pearson, is devoted to the simplification of the method of least squares as applied to the problem of fitting polynomials to data with arguments arranged in an arithmetic progression. The problem resolves itself into the calculation of symmetric determinants and their first minors, the elements of which are the polynomials in $p, S_{n}(p)$, representing the Bernoulli sums,

$$
S_{n}(p)=1^{n}+2^{n}+\cdots+p^{n} .
$$

The mathematical interest of the paper centers about two empirical discoveries, (a) that the symmetric determinants, the elements of which are the Bernoulli sums, are completely factorable into rational factors; (b) that each first minor contains as a factor the first minor of lowest degree in $p$. Although an assumption is made that the data contain an odd number of items, generality is restored to the results by means of a transformation on the moments.

\section{Mr. J. R. Abernethy: A general explicit formula of numerical integration.}

This formula is the most general obtainable for the expression of the approximate value of the definite integral of a function, $f(x)$, as a linear combination of values of the function and its derivatives, containing as special cases all formulas which give exact results if applied to polynomials of degree $n-1$, where $n$ is the number of values appearing in the formula. The choice of values is only limited by the restriction that there be at least $v+1$ values of $f^{z}(x)(z=0,1, \cdots, v ; v=1,2, \cdots, n-1)$. The remainder term is a linear combination of divided differences with repeated arguments.

\section{Professor H. A. Simmons: A calculus of variations problem} whose extremals are parabolas.

Suppose light is traveling in a plane medium whose index of refraction $n$ at any point is directly proportional to the square root of the distance of the point from a fixed line of the plane, and let it be required to find the path in this medium along which light will pass from one point to an other in the shortest time. This problem requires a minimum for the integral

$$
I=\int_{x_{3}}^{4} y^{1 / 2}\left(1+y^{\prime}\right)^{1 / 2} d x \text {. }
$$


(1) The extremals for this problem are the parabolas, the one-parameter family of which through any point $1,\left(x_{1}, y_{1}\right)$, has the equation

$$
y=y_{1}+\left[\left(x-x_{1}\right)^{2}+4 c\left(x-x_{1}\right)\left(y_{1}-c^{2}\right)^{1 / 2}\right] / 4 c^{2} .
$$

(2) The envelope, $G$, of this family is a parabola. (3) Through any point $2,\left(x_{2}, y_{2}\right)$, above $G$ and not in the same vertical line with 1 there pass exactly two parabolas of the family through 1 . (4) One of the two parabolas just mentioned provides a relative minimum for $I$, while the other touches $G$ at a point for which $x_{1}<x<x_{2}$ and therefore does not satisfy Jacobi's condition. (5) There is a discontinuous solution consisting of segments of the straight lines $x=x_{1}, x=x_{2}, y=0$. (6) There is a curve $H$, defined by an elliptic integral, which plays the role here that MacNeish's curve plays in the catenary problem.

\section{Professor V. B. Teach: The Hamilton-Jacobi theory for} the problem of Lagrange in parametric form.

The problem of Lagrange in parametric form is that of finding in a class of $\operatorname{arcs} y_{i}=y_{i}(t), \quad(i=1, \cdots, n)$, passing through two fixed points and satisfying a system of differential equations

$$
\phi_{\beta}\left(y, y^{\prime}\right)=0, \quad(\beta=1, \cdots, m<n-1),
$$

an arc which minimizes the definite integral $\int_{t_{1}}^{t_{2}} f\left(y, y^{\prime}\right) d t$, the symbols $y$ and $y^{\prime}$ representing the sets $\left(y_{1}, \cdots, y_{n}\right)$ and $\left(y_{1}, \cdots, y_{n}\right)$ respectively. In this paper the theory of the parametric problem in $(y)$-space is deduced from the theory of the corresponding non-parametric problem in $(t, y)$-space, a differential equation $\phi\left(y, y^{\prime}\right)-1=0$ defining the parameter $t$ being adjoined to the system $\phi_{\beta}=0$ for the latter problem. First necessary conditions are obtained and shown to be independent of the choice of parameter. The structure of those special Mayer fields in $(t, y)$-space of interest to the parametric problem is next discussed. A canonical form for the differential equations defining extremals and the Hamilton-Jacobi partial differential equation are determined and their properties studied with reference to the theory of fields.

34. Dr. W. D. Baten: Simultaneous treatment of discrete and continuous probabilities by use of Stieltjes integrals.

This paper presents several theorems pertaining to the probability that certain functions lie within certain intervals. The first theorem is a generalization of Markoff's Lemma, which is proved for the discrete and continuous cases by use of the accumulative frequency function and Stieltjes integrals. Tchebycheff's theorem is obtained as a corollary to a very general theorem, the proof of which is based on the first theorem. Other corollaries are given. Three theorems due to Guldberg, concerned with the probability that a non-negative chance variable be less than certain functions of the expected value of the variable, are proved for the discrete and continuous cases by employing accumulative frequency functions and Stieltjes integrals. This is the first time, as far as the writer knows, that the discrete and continuous cases for these theorems have been included in a single proof.

35. Professor C. N. Moore: Gibbs' phenomenon in summable series of Bessel's functions. 
In a previous paper (abstract in this Bulletin, July-August, 1928) the author has shown that the nature of Gibb's phenomenon in the case of convergent series of Bessel's functions can be readily inferred from an asymptotic formula for the coefficients of these series obtained by him in 1911 . In the present paper he shows how a more general asymptotic formula (see Transactions of this Society, vol. 21 (1920), p. 110) serves to answer the the question as to the appearance of Gibbs' phenomenon when the same series are summed by Cesàro means.

\section{Mr. H. C. Chang: Some transformations of linear partial differential equations.}

In the first part of the paper a generalization of Cauchy-Riemann equations given recently by $K$. P. Williams is carried further. A system of two linear homogeneous partial differential equations with constant coefficients involving two independent variables and two functions is considered. It is asked when such a system can be transformed into one equivalent to the Cauchy-Riemann system by subjecting the variables as well as the functions to linear transformations with constant coefficients. It is found that a necessary and sufficient condition is the inequality

$$
4\left(B^{\prime} C^{\prime}-A^{\prime} D^{\prime}\right)(B C-A D)>\left(A D^{\prime}+A^{\prime} D-B C^{\prime}-B^{\prime} C\right)^{2} .
$$

The second part is devoted to a generalization of the problem to the case of four equations involving three variables and three functions. The system into which it is required to transform such a system consists of three equations expressing the fact that the rotation of a vector vanishes and of one equation saying that its divergence vanishes. A necessary and sufficient condition is given here by seven equalities and two inequalities.

\section{Dr. W. T. Reid: Note on an infinite system of linear differential equations.}

In this paper is treated the infinite system of linear differential equations of the first order which we may write in vector form (1): $y^{\prime}=A(x) y$, where $A(x) \equiv\left(A_{i j}(x)\right)$ is an infinite square matrix each of whose elements is Lebesgue summable on $X: 0 \leqq x \leqq 1$ and such that for all real quantities $\xi_{1}, \cdots, \xi_{n}, \eta_{1}, \cdots, \eta_{n}$, and every integer $n$,

$$
\left|\sum_{i=1}^{n} \sum_{i=1}^{n} A_{i j}(x) \xi_{i} \eta_{j}\right| \leqq \phi(x)\left(\sum_{i=1}^{n}\left|\xi_{i}\right|[p /(p-1)][(p-1) / p]\left(\sum_{i=1}^{n}\left|\eta_{j}\right|^{p}\right)^{1 / p}\right.
$$

where $p$ is a real quantity greater than unity and $\phi(x)$ is a summable function on $X$. By definition a solution of (1) is a vector $y(x) \equiv\left(y_{i}(x)\right)$ which satisfies the equation "almost everywhere" on $x$ and such that

$$
\left(\sum_{j=1}^{\infty}\left|y_{i}(x)\right|^{p}\right)^{1 / p}
$$

is bounded uniformly on $X$ by a finite constant. The solutions of (1) are shown to have properties corresponding to those already proved by the author for the case $p=2$ [see this Bulletin, vol. 35 (1929), p. 147 and p. 454].

38. Mr. A. E. Ross: On representation of integers by definite 
ternary quadratic forms whose determinant contains no square factors.

In this paper we study the problem of representation of integers by indefinite ternary quadratic forms. Meyer (Journal für Mathematik, vol. 108 (1891), pp. 125-139) gave a criterion for equivalence of such forms and employed the latter to obtain sufficient (but not necessary) conditions that they represent a given integer $m$. A. W. Markoff (Mémoires, Académie des Sciences, St. Petersbourg, (8), vol. 23 (1909)) constructed a table of indefinite ternary quadratic forms of positive determinants $D \leqq 50$. L. E. Dickson, in an unpublished manuscript, noted regularity in form of the entries of the table and employed a Dirichlet method to obtain complete theorems on representation of integers by forms of determinant $-D$, where $D \leqq 83$ and is either a prime, double a prime, the product of two distinct primes, or double such a product. By Meyer's criterion for equivalence and ideas suggested by Professor Dickson's method this paper solves the problem of representation of integers by indefinite ternary quadratic forms whose determinant is any integer containing no square factors. Theorems proved by Professor Dickson are found to hold for any $D$ of the same type.

39. Professor M. H. Ingraham: Nilpotent and indempotent algebras of infinite order.

Many theorems concerning the nilpotent subalgebras and the idempotent elements of a linear associative algebra of finite order are not available for the infinite case. A definition of transfinite powers of an algebra is given and much of the theory of nilpotent subalgebras reestablished for the case in which the algebra has an index, finite or transfinite. A sufficient condition for the existence of such an index is that the cardinal number of the algebra be an "aleph number." An algebra may not be nilpotent and yet contain no idempotent element. Much of the theory based on idempotent elements may, however, be replaced by one based on idempotent subalgebras. These idempotent subalgebras are of several types corresponding roughly to types of idempotent elements in the finite case.

\section{Professor V. C. Poor: Residues of polygenic functions.}

This paper contains a definition for the residue of a polygenic function; this definition may be thought of as a generalization of the definition for the residue of an analytic function at a point; the contour chosen for the path of integration is a circle of radius $r$; the residue is then defined as the limit of the integral of the function around the circle as $r$ approaches zero. Among the several simple cases treated is the residue of the reciprocal of $a z+b \bar{z}$. Here the poles may be distributed along a straight line through the origin. An application of the definition is also made to the derivation of the "areolar" derivative of Pompieu.

41. Dr. L. M. Biumenthal: Permutable functions in the complex domain.

Pérès has shown that the function $G(x, y)$ permutable with a function 
$F(x, y)$ of the first order and in canonical form can be represented by a transformation $\Omega(\lambda)$ that preserves composition i.e., if $G=\Omega(\lambda), H=\Omega(\mu)$, then $G H=\Omega(\lambda) \Omega(\mu)=\Omega(\lambda \mu)$, where by $G H$ is meant the Volterra product of $G$ and $H$ (Volterra et Pérès, Leģons sur la Composition, p. 58). Those transformations may be written in the form $\left(1^{0}+m\right) \lambda\left(1^{0}+n\right)$, where $m(x, y)$ and $n(x, y)$ are any two functions such that $\left(1^{0}+m\right)\left(1^{0}+n\right)=1^{0}$. For functions $F(x, y)$ of order $n+1$ ( $n$, a positive integer $>1)$ it is found to be of advantage to leave the field of real variables and to consider a function $F(z, w)$ analytic in the complex variables $z, w$. This paper seeks to avoid the restrictions of analyticity and investigates the existence of functions of the first order permutable with a non-analytic function $F$ of order greater than two.

42. Professor I. A. Barnett: Sphere geometry and conformal transformations in function space.

In order to obtain a simple analytic representation of conformal transformations in function space, the author finds it desirable to define sphere coordinates in a manner analogous to that used in $n$-space. He is then able to express the general conformal group in ordinary function space as a linear transformation in sphere function space. As an aid in the discussion of the group properties, the author expresses every conformal transformation as a Fredholm transformation. Infinitesimal conformal transformations are next introduced and it is shown that every infinitesimal conformal transformation generates a one-parameter group of conformal transformations.

43. Professor H. A. Simons: The first variation of a triple integral in the case of variable limits.

This paper contains an extension of a portion of an article by the present author on the first and second variations of a double integral in the case of variable limits (See Transactions of this Society, April, 1926). Here, instead of considering the double integral $\iint_{A_{0}} f(x, y, z, p, q) d x d y$, which was studied in the article just mentioned, we consider the triple integral $\iiint_{V_{0}} f(x, y, z, t, l, m, n)$ $d x d y d z$. We extend our previous methods and obtain analogs of the Euler condition and the transversality condition which were found in the cited article. The hypersurface $t=t(x, y, z)$ of three dimensions, in euclidean $S_{4}$, is given a variation of the type $t=t(x, y, z)+a \tau(x, y, z)$ and these varied hypersurfaces are required to have real closed intersections with a fixed hypersurface $\phi(x, y, z, t)=0$.

44. Professor M. W. Haskell: Autopolar configurations in the plane and in space.

The paper is mainly an account of the author's investigations of methods for deriving curves and surfaces that are autopolar in a finite number of ways. One method is an amplification of Appell's paper in the Nouvelles Annales, (3), vol. 13 (1894). A second method, in three dimensions, employs covariants of transformations of line-coordinates.

45. Professor W. M. Whyburn: Generalized Riccati differential equations. 
The paper considers the set of differential systems defined by the recurrence relations

$F_{0}(Z)=Z(x), F_{i}(Z)=d\left\{F_{i-1}(z)\right\} / d x+F_{i-1}(Z) \cdot Z=f_{i}(x),(i=1,2, \cdots)$, where $Z(x), f_{i}(x)$, and $F_{i}(Z)$ are square matrices of $n^{2}$ elements and $x$ is a real or complex variable. This set of differential equations is shown to be closely related to systems of linear first order differential equations. Important special cases are solved and these solutions shown to represent such important classes of functions as the rational functions, the exponential functions, etc.

\section{Professor W. M. Whyburn: On related difference and} differential systems.

The passage to the limit from difference to differential systems as given by the author (American Journal of Mathematics, vol. 51, pp. 265-286) is shown to be uniform on the interval of definition and to be valid for every method of defining the coefficients of the difference systems so long as these coefficients are uniformly bounded by a summable function and approach the corresponding coefficients of the differential systems almost everywhere. Estimates of the degree of approximation of the solutions of the differential systems by the solutions of the difference systems are immediate consequences of the established uniformity.

\section{Professor E. R. Hedrick: Generalizations of Liouville's} theorem and allied theorems.

In this paper, the author shows first that the usual theorem that the absolute value of a function of a complex variable cannot have a maximum is readily extensible to functions that are non-analytic, provided the jacobian of the corresponding real transformation is different from zero, that is, provided there is no branch point within the region considered. It is then shown that Liouville's theorem can be extended, under the same hypotheses as those usually stated for analytic functions, if the function approaches a definite limit as the variable becomes infinite. The fundamental theorem of algebra, and generalizations of it, follow. Finally, the theorems can all be extended readily to $n$ dimensions; the statements for three dimensions are given.

48. Dr. A. R. Williams: On certain quartic surfaces which are the envelopes of two systems of conicoids.

This paper deals with the envelope of a one-parameter family of ellipsoids or hyperboloids of revolution that have one focus fixed while the other moves on a curve $C$. The characteristics of which the envelope is composed are conics. If the length of the major axis of the conicoid does not vary, the planes of the characteristics are perpendicular to $C$. If $C$ is a circle and the major axis is constant, the envelope is a quartic surface which is also enveloped by two other systems of conicoids of revolution with variable major axes. Such surfaces have useful optical properties.

49. Professor E. T. Bell: Three degeneracies in the theory of ternary quadratic forms. 
All values of $n$ are found for which the number of representations of $n$ in one or other of the three forms $x y+y z+z x, x y+2 y z+2 z x, x y+y z+2 z x$, is a polynomial in the real divisors of $n$ alone. This paper will appear in the Annals of Mathematics.

\section{Professor E. T. Bell: A triple of ternary quadratic forms.}

Beyond pure quadratic ternaries, for numbers of representations $a x^{2}+b y^{2}$ $+c z^{2}$, only one ternary quadratic, namely, $x y+y z+z x$, has been discussed, notably by Liouville, Bell, Mordell, and Gage. In this paper is presented a complete discussion concerning numbers of representations in each of the three closely connected forms $(1,1,1),(1,1,2),(1,2,2)$, where $(a, b, c) \equiv a x y$ $+b y z+c z x$.

51. Professor E. T. Bell: On certain quinary and senary quadratic forms.

Certain general principles, too detailed for concise abstract, are laid down for enumerations of representations in $n$-ary quadratic forms and are applied to the cases $n=5$, 6 . In general, an odd and its consecutive even number of variables are treated together in the theory, which is new and which has but slight connection with classical theories.

\section{Professor D. N. Lehmer: On Franklin magic squares.}

The author has found a generalization of Franklin's method of constructing magic squares of even order which applies to oddly even as well as to evenly even squares. Enumeration formulas have been developed. The number of $4 \times 4$ squares has been determined to be at least 6144 instead of 4352 as determined by Andrews. They all come from four types of Franklin squares.

53. Mr. W. H. Ingram: A system of integral equations implying and implied by a system of first order linear differential equations.

Bounitzky has shown how, given a system of first order linear differential equations satisfying a set of boundary conditions for certain values of a parameter, a system of integral equations of the second kind can be constructed whose solutions satisfy the differential equations and the boundary conditions. It has not been shown that no other system of integral equations could have a similar property. It is now shown that the system, $v^{\prime}+(r(x)+\lambda l(x)) u=0$, $\left.u^{\prime}+s(x)+\lambda c(x)\right) v=0$, with the boundary conditions $a_{1} v(0)+b_{1} u(0)=0$, $a_{2} v(1)+b_{2} u(1)=0$, implies and is implied by the system of integral equations

$$
\begin{gathered}
v=\lambda \int_{0}^{1}\left\{K_{11}(x, \xi) v(\xi)+K_{12}(x, \xi) u(\xi)\right\} d \xi, \\
u=\lambda \int_{0}^{1}\left\{K_{21}(x, \xi) v(\xi)+K_{22}(x, \xi) u(\xi)\right\} d \xi,
\end{gathered}
$$

where $K_{11}(x, \xi)=c(\xi) G_{11}(x, \xi)$, etc., in which the $G$ 's are Green's functions. 
54. Mr. W. H. Ingram: Systems of integral equations and their Fredholm solutions.

The system of integral equations of the previous paper has, formally, two solutions of Fredholm type for $v$ and two for $u$. From the fact that the Green's functions can be expressed as the products of fundamental solutions (Grundlösungen of the Schmidt-Hilbert theory) in the form $G_{11}(x, y)=-v^{* *}(y) v^{*}(x)$, $G_{12}(x, y)=-u^{* *}(y) v^{*}(x)$, for $0 \leqq x<y$, or $G_{11}(x, y)=-v^{*}(y) v^{* *}(x), G_{12}(x, y)$ $=-u^{*}(y) v^{* *}(x)$, for $y \leqq x \leqq 1$, and from the relations of symmetry $G_{11}(x, y)$ $=G_{11}(y, x), G_{12}(x, y)=-G_{21}(y, x), G_{22}(x, y)=G_{22}(y, x)$, it appears that when constants $t_{0}, t_{1}$ can be so chosen that we have simultaneously $u^{* *}\left(t_{0}\right) l\left(t_{0}\right)$ $=v^{* *}\left(t_{1}\right) c\left(t_{1}\right), u^{*}\left(t_{0}\right) l\left(t_{0}\right)=v^{*}\left(t_{1}\right) c\left(t_{1}\right)$, then the solutions for $u$ and $v$ respectively are identical.

55. Mr. W. H. Ingram: Application of the theory of integral equations to the theory of the electrical transmission line.

The differential equations are those of the first paper. The Green's functions represent current and potential due to two types of source physically representable by voltaic cells connected to the line by insertion in series at a point or by parallel connection to ground at a point; they are, therefore, experimentally tabulable. The problem of transient oscillations presents certain difficulties due to the fact that the system. of differential equations is not self-adjoint. When the line is dissipationless, any given initial current and potential distributions $U$ and $V$ have the expansions $\sum a_{i} l^{1 / 2}\left(u_{i}+\bar{u}_{i}\right)$, $\sum a_{i} c^{1 / 2}\left(v_{i}+{ }_{i}\right)$ respectively, where the $a_{i}$ 's can be determined to minimize the quantity

$$
N=\int_{0}^{1}\left\{\left[U-\sum a_{i} l^{1 / 2}\left(u_{i}+\bar{u}_{i}\right)\right]^{2}+\left[V-\sum a_{i} c^{1 / 2}\left(v_{i}+\bar{v}_{i}\right)\right]^{2}\right\} d x .
$$

In the presence of dissipation ( $r$ and $s$ not zero), a similar expansion is obtainable which minimizes (maximizes) the indefinite form

$$
M=\int_{0}^{1}\left\{\left[U-\sum a_{i} l^{1 / 2}\left(u_{i}+\bar{u}_{i}\right)\right]^{2}-\left[V-\sum a_{i} c^{1 / 2}\left(v_{i}+\bar{v}_{i}\right)\right]^{2}\right\} d x .
$$

56. Professor Raymond Garver: On the transformation which reduces the Brioschi normal quintic to a general principal quintic.

If the transformation

$$
Y=\frac{\lambda+\mu w}{Z^{-1} w^{2}-3}
$$

is applied to the Brioschi quintic

$$
w^{5}-10 Z w^{3}+45 Z^{2} w+k=0 \text {, }
$$

the transformed equation $F(Y)=0$ is of the so-called principal form, lacking terms in $Y^{4}$ and $Y^{3}$. Further, $F(Y)=0$ can be identified with any principal quintic, with certain minor exceptions, by properly choosing $\lambda, \mu, Z$. The importance of the transformation depends on this fact, since it follows almost at once that any sufficiently general quintic can be reduced to the Brioschi form. This paper gives a method of forming $F(Y)=0$ which seems simpler 
than the methods which have been given previously, and which allows the whole topic to be presented in a very convenient form.

57. Professor Raymond Garver: Concerning polynomial functions with certain properties.

Brioschi has shown the existence, for the general $n$th degree equation, $n$ odd, of $(n-1) / 2$ polynomial functions which possess certain properties making them of value in a number of transformations of equations. His proof, however, is long and yet incomplete. This paper presents what seems to be a more satisfactory proof. The case where $n$ is even is also considered, and it is shown that $n / 2$ functions with the desired properties cannot, in general, be set up.

58. Professor Florian Cajori: Newton's idea of God in the different editions of the Principia.

Although the first edition of the Principia contains no direct expression of the nature of God, Newton later stated that he hoped its contents would lead to a belief in the Deity. The Principia was criticised, on theological grounds, by Bishop Berkeley and by Leibniz. In the second edition Newton added a general scholium giving his idea of God as seen from His works. Interpolations in the third edition stressed the idea that our notions of God are taken more especially from the ways of mankind.

59. Dr. Gordon Pall (National Research Fellow): On the representations of a constant times a square as the sum of an odd number of squares.

Stieltjes and A. Hurwitz found expressions as simple divisor functions for the number $r\left(n^{2}\right)$ of the representations of $n^{2}$ as the sum of $s$ squares, $s=3$ or 5 . This paper gives a general formula for the ratio $r\left(n^{2} c\right) / r(c)$ as a function of divisors, when $s=3,5,7$ and when $s=11, c \equiv 7(\bmod 8)$. When $c=1, s=3,5$, this reduces to Hurwitz's results.

60. Dr. B. W. Jones (National Research Fellow): On certain irregular ternary forms with cross products.

In this paper the writer finds that in the neighborhood of 200 reduced positive primitive forms $f=a x^{2}+b y^{2}+c z^{2}+2 r y z$ are regular, where $b, c$, and $2 r$ have no factor greater than 2 in common and $r$ is odd if $b$ and $c$ are even. This is equivalent to finding what forms $x^{2}+d y^{2}+p a z^{2}$ and $x^{2}+d y^{2}+2 p a z^{2}$ are regular as to multiples of $p$ or $2 p$ respectively, where $(-d \mid p)=1, p$ is a prime, and, in the second case, $d \equiv 3(\bmod 4)$.

61. Dr. Clifford Bell: In- and circumscribed sets of planes to space curves.

In this paper the number of in- and circumscribed sets of osculating planes to the various 3 -space rational quartic curves is determined. It is also shown that there are $4 n$ unclosed sets of three osculating planes to the cuspidal quartic, each set of which has its point of intersection on the surface $f(x, y, z, w)=0$ 
of order $n$. The points of contact of the first, second, and third osculating planes are represented respectively by $A_{i}, B_{i}, C_{i}$, the other intersection of the third plane being represented by $D_{i}$, and the point on the surface $f(x, y, z, w)=0$ at which the three planes intersect is represented by $P_{i},(i=1,2, \cdots 4 n)$. It is proved that the surface $f(x, y, z, w)=0$ can be deformed by a simple magnification of $x, y, z$, and $w$ in such a way as to contain all the points $A_{i}$, and likewise for the points $B_{i}, C_{i}$, and $D_{i}$. This is also extended to the analogous curve in $n$-space.

62. Dr. H. T. Engstrom (National Research Fellow): On the ideal theory of Zolotarev.

This paper is a commentary on the theory of Zolotarev based on a translation from the Russian of an exposition by Tchebotarev. The common criticisms of the theory on the grounds of incomplete generality are probably based on the inadequate account in the Fortschritte der Mathematik and are unjustified.

63. Dr. J. D. Elder: Fourier expansions of doubly periodic functions of the third kind.

A method, due to Appell, is applied to the determination of the Fourier expansion of the function $F(z)=\theta_{0} n_{0}(z) \theta_{1} n_{1}(z) \theta_{2} n_{2}(z) \theta_{3} n_{3}(z)$, where the $n$ 's are integers such that $n_{0}+n_{1}+n_{2}+n_{3}<0$. Sixteen sub-cases are considered, which arise from the possible parities of the $n$ 's. For each of these, an explicit formula of expansion is found, valid for general values of the $n$ 's of appropriate parities.

64. Dr. Morgan Ward: The characteristic number of a sequence of integers satisfying a linear recursion relation.

The author shows that if $(A)_{n}$ is any sequence of integers satisfying $X_{n+3} \equiv P X_{n+2}-Q X_{n+1}+R X_{n}(\bmod m)$, and if $m=p_{1}{ }^{a_{1}} \cdots p_{r}{ }^{a_{r}}$ is the decomposition of $m$ into prime factors, then the characteristic number of $(A)_{n}$ modulo $m$ is the L. C. M. of its characteristic numbers moduli $p_{1}{ }^{a_{1}}, \cdots, p_{r}{ }^{{ }}$. If $m=p^{a}$, the characteristic number is of the form $p \mu^{b}$, where $\mu$ is the characteristic number of $(A)_{n}$ modulo $p$, and $b \leqq a-1$. The determination of $\mu$ is equivalent to the unsolved problem of determining the period of a given mark in a given Galois field. The results obtained may easily be extended to the general case of a recursion relation of order $r$. (For the definition of the characteristic number, see Carmichael, American Mathematical Monthly, March, 1929.)

\section{Professor P. H. Daus: Note on a table of linear forms.}

In constructing his table of linear forms, used in making his factor stencils, D. N. Lehmer used the fact that if $r_{1}$ and $r_{2}$ are numbers in a linear form, corresponding to a given $D$, then $r_{1} r_{2}$ is also a number of the form. This paper is concerned with the question of finding an $r$, if possible, for a given $D$, such that all numbers of the form can be obtained from $r$. It is shown that if $\pm D$ is a prime or twice a prime, such an $r$ can readily be found, using available tables 
of primitive roots and the quadratic reciprocity law. By means of the rules established, it is possible to construct a compact table of linear forms.

66. Professor J. V. Uspensky: A simple proof of the theorem on the limit of probability.

The fundamental theorem usually called "the theorem on the limit of probability" was proved under very general conditions by Liapounoff. This remarkable proof is based on a very ingenious device consisting in the introduction of an auxiliary contin tous variable. This auxiliary variable does not appear explicitly in the simplified proof presented in this paper, which makes it comparatively easy and short. 